\title{
Configuration of Neural Networks for the Analysis of Seasonal Time Series
}

\author{
T.Taskaya-Temizel and M.C.Casey \\ t.taskaya,m.casey@surrey.ac.uk \\ Department of Computing, School of Electronics and Physical Sciences, \\ University of Surrey, Guildford GU2 7XH,UK
}

\begin{abstract}
Time series often exhibit periodical patterns that can be analysed by conventional statistical techniques. These techniques rely upon an appropriate choice of model parameters that are often difficult to determine. Whilst neural networks also require an appropriate parameter configuration, they offer a way in which non-linear patterns may be modelled. However, evidence from a limited number of experiments has been used to argue that periodical patterns cannot be modelled using such networks. In this paper, we present a method to overcome the perceived limitations of this approach by determining the configuration parameters of a time delayed neural network from the seasonal data it is being used to model. Our method uses a fast Fourier transform to calculate the number of input tapped delays, with results demonstrating improved performance as compared to that of other linear and hybrid seasonal modelling techniques.
\end{abstract}

\section{Introduction}

In time series analysis, the recognition of patterns is important to facilitate the estimation of future values. This is especially evident for financial time series forecasting, where techniques such as technical and regression analyses have been developed that rely upon the identification of different temporal patterns [1]. In particular, regression analysis, whose application area is not only limited to financial forecasting [2-4], relies on the identification of patterns within the series, such as trend and seasonality. These patterns can be modelled using statistical techniques, such as autoregressive (AR) variants, but constructing such models is often difficult. Whilst the application of neural networks to time series analysis remains controversial $[1,4]$, they appear to offer improved performance, for example, when used in hybrid models [5]. In hybrid models applied to seasonal data series, seasonality is first decomposed using techniques such as linear filters [1]. One such method is the application of the autocorrelation function to determine the input lags used to build a linear AR model, and in particular only the significant lags are selected [3]. However there is evidence to suggest that using just these selected lags does not give optimal linear models [6]. Furthermore, it is unclear whether the lags obtained from the autocorrelation function are sufficient for use in a non-linear model, such as a neural network. In this paper 
we describe a method to configure a neural network to model time series that exhibit cyclic behaviour using a more appropriate selection of input lags. When applied to a time delayed neural network (TDNN), our method demonstrates similar performance compared to more complex hybrid modelling techniques.

In statistics, periodical variations are treated in two different ways. First, if periodical patterns change stochastically during time, one can apply seasonal differencing to eliminate seasonality. Second, if behaviour of the periodicity is deterministic, models can be applied by taking into account the type of the variation, such as whether the changes are in additive or multiplicative form. Linear processes such as AR models, seasonal AR models and the Holt-Winters method are among the few seasonal modelling techniques that have proven successful [1, 3]. However, to model non-linear patterns, appropriate non-linear techniques are required, such as neural networks.

Non-linear neural networks are capable of extracting complex patterns in time series successfully to some degree $[2,7]$, although identifying whether nonlinear models are required remains difficult [1]. A well-known technique to perform temporal processing is to use memory of past input and activation values within the network to allow it to identify temporal patterns. These TDNN models $[7,8]$ are widely used because of their simplicity, either on their own [9-11], or in hybrid models such as with an autoregressive integrated moving average model (ARIMA) $[5,12,13]$. Nevertheless, it has been reported that such hybrids do not necessarily outperform single models [11].

The limitations of neural networks are essentially an inability to cope with changes in mean and variance [9], which can be attributed, for example, to trend and exponential seasonality. The mean and variance of a series may be stabilised using techniques such as differencing and the Box-Cox transformation [14]. Similarly, it has been argued that periodical patterns should be removed prior to modelling with a neural network $[12,13,15,16]$. However, it has been shown that certain periodical patterns can be successfully modelled using neural networks if the network is configured appropriately, and the time series preprocessed to stabilise the mean [10].

The studies so far have focused on model selection tools designed for building linear AR models, which are then used to construct a TDNN for cyclic series $[9,12]$. For example, Cottrell [9] used Akaike and Bayesian Information Criteria to find an optimum TDNN. For the Sunspot data, their results suggested an architecture with four input delays and four hidden neurons. Despite this, the Sunspot data exhibits an 11 year cycle, and results suggest that a minimum lag of 11 is required (for example, $[2,17]$ ). In contrast to Cottrell's approach, Zhang and Qi [12] used the autocorrelation function to configure a TDNN. First, they obtained the significant lags within the series based on the an analysis of the autocorrelation. Then they employed these lags to construct the input delays of TDNN. In this paper we describe a method, which is an extension of that described in [10], for selecting the input delays using a fast Fourier transform, comparing our results with Zhang and Qi's. 


\section{Configuring TDNN for Seasonal Time Series}

A TDNN is a variant of the multi-layer perceptron in which the inputs to any node $i$ can consist of the outputs of the earlier nodes generated between the current step $t$ and step $t-d$, where $d \in Z^{+}$and $\forall d<t$. Here, the activation function for node $i$ at time $t$ is:

$$
y_{i}(t)=f\left(\sum_{j=1}^{M} \sum_{d=1}^{T} w_{i j}(t-d) y_{j}(t-d)\right)
$$

where $y_{i}(t)$ is the output of node $i$ at time $t, w_{i j}(t)$ is the connection weight between node $i$ and $j$ at time $t, T$ is the number of tapped delays, $M$ is the number of nodes connected to node $i$ from preceding layer, and $f$ is the activation function, typically the logistic sigmoid. In this paper, we consider the case when we have tapped delays in the input layer only: an IDNN.

In order to set the number of delays in the input layer, we obtain the cycle information from the training data using a fast Fourier transform, which sometimes gives similar results to that of applying the autocorrelation function, but is more convenient to use within a systematic method. In the choice of the cycle information, we consider the dominant cycles within the data as determined by the outliers in the amplitude response. Then we construct the TDNN with the number of tapped delays $I L$ equal to each of the extracted dominant cycles, choosing the final configuration based upon the best performing network on the validation data set. Specifically:

1. Given a time series $\left\{x_{i}\right\}_{i=1}^{K}$, create training $\left\{x_{i}\right\}_{i=1}^{T R}$, validation $\left\{x_{i}\right\}_{i=T R+1}^{V L}$ and test data sets $\left\{x_{i}\right\}_{i=V L+1}^{K}$, where $i$ is the time index.

2. Stabilise the mean of the series by computing the first-order difference.

$$
x_{i+1}^{\prime}=x_{i+1}-x_{i}
$$

where $x^{\prime}$ is the stabilised time series.

3. Estimate the number of input tapped delays $I L$ using the dominant cycle information in the differenced series:

(a) Compute the fast Fourier transform of $\left\{x_{i}^{\prime}\right\}_{i=1}^{V L}$ :

$$
X_{i}=\sum_{j=1}^{V L} x_{j}^{\prime} w_{V L}^{(j-1)(i-1)}
$$

where $w_{V L}=e^{(-2 \pi i) / V L}$ is a $V L^{t h}$ root of unity.

(b) Let $R_{i}^{0}=\left|X_{i}\right|$ be the amplitude response of $X_{i}$.

(c) Discard the periods that are greater than $V L / 2$.

(d) Set $j=1$.

Let $S$ be a set and set $S=\oslash$.

Let $P_{i}$ be the set of periods. 
While not finished

Compute the mean $\mu_{j-1}$ and standard deviation $\sigma_{j-1}$ of $R_{i}^{j-1}$

Extract the outliers $P_{i}$, where $R_{i}^{j-1}>\mu_{j-1}+3 \sigma_{j-1}$

Set $S=S \cup P_{i}$.

Set $R_{i}^{j}$ be the amplitude response without the outliers $P_{i}$.

Set $j=j+1$.

If $P=\oslash$ exit loop.

End while

(e) Set the number of input tapped delays $I L$ to the closest integer value \pm 2 [12] for each period within $S$. The best number will be selected experimentally from these according to the test set performance.

4. Restrict the number of nodes in the output layer to unity and set the hidden layer size

$$
H \leq \frac{(I L+1)}{2}
$$

5. Normalize the series using the z-score to improve training in the network.

$$
z_{i}=\frac{x_{i}^{\prime}-\overline{x^{\prime}}}{\sigma_{x^{\prime}}}
$$

Note that the outliers $P$ in Step 3d are rounded integer periods.

\section{Experiments and Results}

To evaluate this method, we chose four industrial production series (from Federal Reserve Board [18]): consumer goods (starting January 1970), durable goods (starting January 1947), fuels (starting January 1954), and total industrial production (starting January 1947)and five U.S. Census Bureau series [19] (starting January 1992): retail, hardware, clothing, furniture, and bookstore, all ending in December 2001 [12]. Each of these monthly data sets exhibit strong seasonalities that are difficult to predict.

In order to compare the selected architectures using the method described with the performance of a TDNN in general, we conducted a number of experiments with network configurations of $2 i: 2 j: 1$, where $1 \leq i \leq 33$ and $1 \leq j \leq 16$, a total of 528 networks. Each network was configured to use a hyperbolic tangent activation function for the hidden layer and a linear function for the output layer. Training was performed using the gradient descent algorithm for a maximum of 20,000 epochs, with initial learning rate parameter $\lambda=0.1$, increased by $1.05 \%$ if the training error decreased, otherwise decreased by $0.7 \%$, if the training error increased by over $4 \%$. Each configuration was tested with 30 different random initial conditions to provide an average root mean square error (RMSE). The testing data set was used to determine which was the best architecture once training was complete. 


\subsection{Results}

For each of the selected input delay sizes $I L$ per data set, Table 1 shows the architecture selected by our algorithm and the best performing network within the trials. In five data sets (FR Fuels, FR Total Production, USBC Bookstore, USBC Clothing, USBC Retail, USBC Hardware), we obtained the best performing architectures among our trials using our method. In the other four data sets, the method did not pick up the best performing architecture. However, the selected architectures were amongst the top ten within the trials.

Table 1. The selected method's performances compared with the performances of the best network configuration obtained from the trials. Bold values show the correctly identified TDNN configurations by the algorithm. Our algorithm finds the best configurations on five out of nine data sets.

\begin{tabular}{|c|c|c|c|c|}
\hline \multirow{2}{*}{ Data Sets } & \multicolumn{2}{|c|}{ Selected Model } & \multicolumn{2}{|c|}{ Best Model } \\
\hline & Config & RMSE & Config & RMSE \\
\hline FR Consumer Goods & 16:02:01 & $1.25 \pm 0.17$ & 24:02:01 & $1.07 \pm 0.89$ \\
\hline FR Durable Goods & 14:06:01 & $3.15 \pm 0.51$ & 12:16:01 & $2.91 \pm 0.34$ \\
\hline FR Total Production & 42:02:01 & $0.99 \pm 0.05$ & 42:02:01 & $0.99 \pm 0.05$ \\
\hline FR Fuels & 30:02:01 & $1.73 \pm 0.12$ & 32:02:01 & $1.64 \pm 0.13$ \\
\hline USBC Bookstore & 12:02:01 & $91.51 \pm 10.40$ & 12:02:01 & $91.51 \pm 10.40$ \\
\hline USBC Clothing & 14:02:01 & $378.71 \pm 68.29$ & 14:02:01 & $378.71 \pm 68.29$ \\
\hline USBC Furniture & 14:02:01 & $175.02 \pm 10.57$ & 48:02:01 & $161.09 \pm 22.63$ \\
\hline USBC Retail & 14:02:01 & $634.72 \pm 30.97$ & 14:02:01 & $634.72 \pm 30.97$ \\
\hline USBC Hardware & 12:04:01 & $37.97 \pm 9.34$ & 12:04:01 & $37.97 \pm 9.34$ \\
\hline
\end{tabular}

In order to understand whether the method selects near optimum parameters for the TDNN, we compared the results given in Table 1 with those for each of the 528 networks constructed over 30 trials. Figure 1 shows the results for each of these networks for the FR total production data set. Part (a) shows the dominant cycles determined by the method, part (b) shows the performance of each of the 528 networks, with the number of neurons within the hidden layer on the $x$-axis, and the number of input tapped delays on the $y$-axis. The shading shows the average RMSE, with the dark areas showing the lowest values. Here we see that the best performing architecture is that with 2 hidden neurons and approximately 42 input tapped delays. This corresponds well with our method, which selects 43 as one of the dominant periods, and with the best performing architecture using 42 input delays, within our bounds of \pm 2 , as suggested by [12]. Apart from FR durable goods, a TDNN has optimal performance when the hidden layer size is set between 2 and 4 . We can therefore see that our method provides a way in which the dominant cycle information can be successfully used to construct a near-optimum TDNN to model seasonal time series.

In Table 2, we compared our best fit results among the selected networks with the best fit of the hybrid ARIMA models constructed by Zhang and Qi [12]. 
(a)

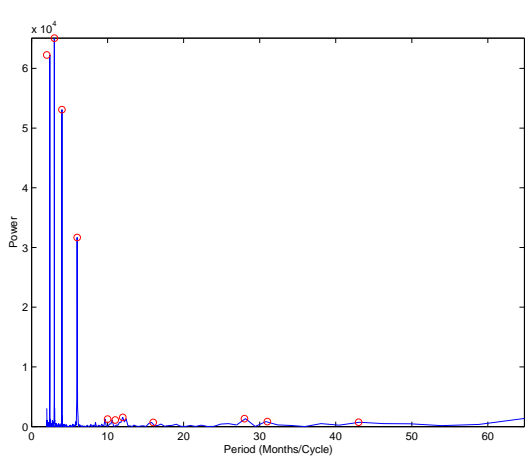

(b)

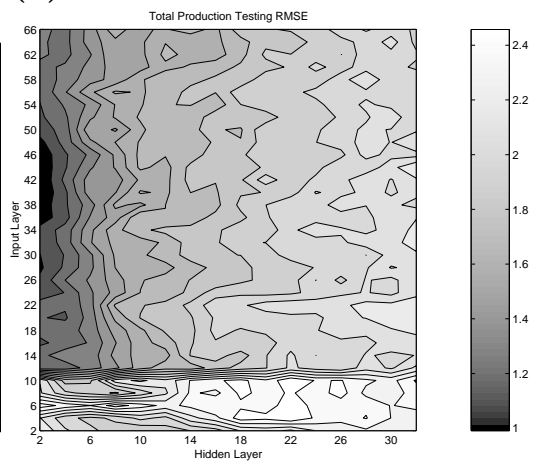

Fig. 1. (a) shows the dominant cycles, which are automatically picked up by the algorithm, (b) shows the error surface of the TDNN on 'Total Production' time series.

For the hybrid architecture, our TDNN models outperformed in six data sets (FR Durable, FR Total Production, USBC Bookstore, USBC Clothing, USBC Retail, USBC Hardware). Zhang and Qi constructed the networks by taking into consideration the correlation structure of the series. Based on the outcome of their analysis, they considered ten time lags: 1-4, 12-14, 24, 25, and 36, where 12,24 , and 36 months apart are highly correlated. The number of hidden nodes varied between 2 and 14 with an increment of 2 . For example, the best neural network configuration for the USBC retail series was $36: 12: 1$, where 36 shows the maximal lagged term. Their findings showed that the input layer should comprise at least a maximal lag of 12 ( 5 input nodes) for all series. More specifically, they reported that for the detrended data using polynomial fitting, the maximal lags were identified as 13 for USBC retail, 36 for FR consumer goods, and 14 for durable goods. For the detrended and deseasonalised data, they used a maximal lag of 1 for USBC retail, 4 for FR consumer goods and 4 for durable goods. They concluded that they found large discrepancies among the hidden layer nodes. However, we found that neural networks with a small number of hidden nodes perform significantly better than ones with a large number of hidden nodes in our earlier study [11]. We observed that the networks trained with continuous lag information outperform networks without.

\section{Conclusion}

We have described an algorithm that can be used to find the optimum TDNN configuration for modelling seasonal time series. The method described selects a number of candidate architectures that include those that are the best performing for this technique compared to existing results. The results also demonstrate that a TDNN model can produce comparable performance to other hybrid models. One advantage with this approach is that the performance of an ARIMA 
Table 2. The best fit selected architectures compared with the performance of TDNN and hybrid architecture constructed by Zhang and Qi [12]. Bold values indicate the minimum RMSE obtained per data set. Our TDNN architectures outperform in all data sets compared to Zhang and Qi's TDNN architectures. They also outperform on five out of nine data sets compared to their hybrid architectures.

\begin{tabular}{|l|c|r|c|c|}
\hline Data Sets & Selected Model & RMSE & TDNN [13] & ARIMA-NN[13] \\
\hline FR Consumer Goods & $18: 04: 01$ & 0.91 & 1.48 & $\mathbf{0 . 6 8}$ \\
\hline FR Durable Goods & $12: 02: 01$ & $\mathbf{2 . 1 6}$ & 5.98 & 3.63 \\
\hline FR Total Production & $42: 02: 01$ & $\mathbf{0 . 7 7}$ & 1.62 & 0.85 \\
\hline FR Fuels & $30: 04: 01$ & 1.30 & 1.83 & $\mathbf{0 . 8 1}$ \\
\hline USBC Bookstore & $12: 02: 01$ & $\mathbf{7 1 . 1 0}$ & 170.49 & 88.74 \\
\hline USBC Clothing & $14: 02: 01$ & $\mathbf{2 7 7 . 3 4}$ & 1117.72 & 315.43 \\
\hline USBC Furniture & $14: 06: 01$ & 144.32 & 226.68 & $\mathbf{9 9 . 4 5}$ \\
\hline USBC Retail & $14: 02: 01$ & $\mathbf{5 4 6 . 5 8}$ & 1785.77 & 975.55 \\
\hline USBC Hardware & $12: 02: 01$ & $\mathbf{1 9 . 7 6}$ & 105.12 & 49.17 \\
\hline
\end{tabular}

neural network hybrid is likely to degrade due to overfitting [11]. In this case it therefore appears that using relatively simple models can improve performance.

One restriction to our method is that it can only model stationary seasonal time series. For example, our method cannot model series in which the amplitude of the cycle increases constantly over time. To be able to model such a series with neural networks, first either the series should be stabilised using an appropriate transformation, or a seasonal AR model should be used. Furthermore, the poorer performance of our method on three data sets (FR Consumer Goods, FR Fuels and USBC Furniture) requires further investigation to determine whether there are any particular characteristics of these that affects our method.

In evaluating the method through comparison of different network configurations, we note that as the number of free parameters increases in the network (the number of input delays and hidden neurons), that the model is likely to overfit both to the training and validation data sets giving poor generalisation. Experimentally this tells us that the input layer size should be set to less than ten percent of the total data set size in order to achieve improved results, but further investigation is required to formalise this. Similarly, we also note that a TDNN generally has optimal performance on the selected data sets when the hidden layer size is set between 2 and 4 , commensurate with our previous work $[10,11]$.

\section{Acknowledgements}

We are grateful to the Fingrid project [RES-149-25-0028], which provided us a Grid environment comprising 24 machines to run our simulations. 


\section{References}

1. Chatfield, C.: The Analysis of Time Series. Sixth edn. Texts in Statistical Science. Chapman \& Hall, USA (2004)

2. Rementeria, S., Olabe, X.B.: Predicting sunspots with a self-configuring neural system. In: Proceedings of the 8th Conference on Information Processing and Management of Uncertainty in Knowledge-Based Systems (IPMU 2000). (2000)

3. Box, G., Jenkins, G.: Time Series Analysis: forecasting and control. Texts in Statistical Science. Holden Day (1970)

4. Faraway, J., Chatfield, C.: Time series forecasting with neural networks: A comparative study using the airline data. Applied Statistics 47 (1998) 231-250

5. Zhang, G.P.: Time series forecasting using a hybrid ARIMA and neural network model. Neurocomputing 50 (2003) 159-175

6. Rao, T.S., Sabr, M.M.: An introduction to bispectral analysis and bilinear time series models. Lecture Notes in Statistics 24 (1984)

7. Wan, E.: Time series prediction by using a connectionist network with internal delay lines. In Weigend, A., Gershenfeld, N., eds.: Time Series Prediction: Forecasting the Future and Understanding the Past. SFI Studies in the Sciences of Complexity, Addison Wesley (1994) 1883-1888

8. Waibel, A., Hanazawa, T., Hinton, G., Shikano, K., Lang, K.J.: Phoneme recognition using time-delay neural networks. IEEE Transactions on Acoustics, Speech and Signal Processing 37 (1989) 328-339

9. Cottrell, M., Girard, B., Girard, Y., Mangeas, M., Muller, C.: Neural modeling for time series:a statistical stepwise method for weight elimination. IEEE Transactions on Neural Networks 6 (1995) 1355-1364

10. Taskaya-Temizel, T., Casey, M.C., Ahmad, K.: Pre-processing inputs for optimallyconfigured time-delay neural networks. IEE Electronic Letters 41 (2005) 198-200

11. Taskaya-Temizel, T., Ahmad, K.: Are ARIMA neural network hybrids better than single models? In: Proceedings of International Joint Conference on Neural Networks (IJCNN 2005), Montréal, Canada (2005) to appear

12. Zhang, G.P., Qi, M.: Neural network forecasting for seasonal and trend time series. European Journal of Operational Research 160 (2005) 501-514

13. Tseng, F.M., Yu, H.C., Tzeng, G.H.: Combining neural network model with seasonal time series ARIMA model. Technological Forecasting and Social Change 69 (2002) 71-87

14. Box, G.E.P., Cox, D.R.: An analysis of transformations. JRSS B 26 (1996) 211-246

15. Virili, F., Freisleben, B.: Nonstationarity and data preprocessing for neural network predictions of an economic time series. In: Proceedings of International Joint Conference on Neural Networks (IJCNN 2000), Como, Italy (2000) 5129-5136

16. Nelson, M., Hill, T., Remus, W., O'Connor, M.: Time series forecasting using neural networks: Should the data be deseasonalized first? Journal of Forecasting 18 (1999) 359-367

17. Weigend, A., Gershenfeld, N.A., eds.: Time Series Prediction: Forecasting the Future and Understanding the Past. Addison-Wesley, Reading, MA (1993)

18. Federal Reserve Time Serial Data. http://www.federalreserve.gov/releases/ G17/table1_2.htm (2005) Last accessed: Jan.2005.

19. U.S. Census Bureau Monthly Trade and Food Services Data Sets. http://www . census.gov/mrts/www/mrts.html (2005) Last accessed: Jan.2005. 